\title{
European Manifesto: Basic Standards of Healthcare for People with Intellectual Disabilities
}

\author{
Frans A Scholte(I)
}

$\mathrm{P}$ eople with intellectual disabilities can have health needs that differ from those of the general population. They have more medical problems and the morbidity pattern is different and often related to the cause of the disabilities. Communicational difficulties and behaviour can disturb the diagnostic process and treatment is not always possible as for anybody else. People with intellectual disabilities who live in society with a minimum of support often have an unhealthy lifestyle and participation on screening programmes is poor. A lack of financial means and bad housing can also contribute to a life with more health risks.

Health care has to pay attention to these differences and special health needs. In 2003, at a conference in Rotterdam, The Netherlands, standards of health care for people with intellectual disabilities were formulated. These standards were meant to be useful in all countries with different health care systems. They are based on the idea that mainstream health services should be equipped to support the specific health needs of intellectually disabled patients. However, health practitioners who are involved with only a few individuals with intellectual disabilities, cannot be expected to have specialised knowledge about the specific health problems of people with intellectual disabilities. Specialists are therefore needed to support them.

In developing countries specialist medical care is not the first priority in the lives of people with intellectual disabilities. You have to think first on the recognition of their human rights, which include the right to equal participation in society. In a lot of countries this will require the improvement of housing and hygiene, schooling or training and the possibility to work or to participate in other meaningful daily activities. Another consequence of the acceptation of equal rights for persons with intellectual disabilities is the willingness of mainstream health professionals to provide adequate health care for these individuals.

An organisation that is focussed on the improvement of medical care for people with intellectual disabilities in European countries is MAMH which stands for Medical Aspects of Mental Handicap.

\section{European Association of Intellectual Disability Medicine}

MAMH was founded in 1991 in Noordwijkerhout in The Netherlands, where the Dutch Association celebrated its $10^{\text {th }}$ anniversary with an international conference. Doctors from different countries, who were involved in the care for people with intellectual disabilities, met each other and considered it important to exchange knowledge and experiences in the medical care for these people. They decided to establish an organisation MAMH, later renamed as European Association of Intellectual Disability Medicine.

The objectives of MAMH are:

- To collect available knowledge and to exchange information regarding the causes, prevention and

(I) Physician for people with intellectual disabilities, president of European Association of Intellectual Disability Medicine. 
medical management of different types of intellectual disability.

- To stimulate and support applied scientific research, often in collaboration with other scientific disciplines.

- To initiate, support and promote education in the medical care of persons with intellectual disability.

- To give advice, where necessary, on medical care to national and international organisations in the care of persons with an intellectual disability.

These objectives are rather ambitious for a small organisation like $\mathrm{MAMH}$, with members who are always very busy with their own work. Nevertheless, we made progress.

MAMH organised congresses in Rome, Manchester, Debrecen and Lahti. MAMH was guest in Kassel when German colleagues established the Kasseler Erklärung. Dr. Sylvia Carpenter, former president of MAMH, gave twice an overview of post-graduate training programmes for doctors in intellectual disability medicine in European countries.

\section{European Manifesto}

As mentioned above it is well known that people with intellectual disabilities have health needs that differ from the general population. Morbidity rates are higher and the morbidity pattern is different. There are also syndrome related health problems. In the care for these people there are often communication problems. Mainstream health services have often little knowledge of these differences, and, which is even worse, have little interest in the special needs of this group of patients. Therefore, the quality of medical care for people with intellectual disabilities in European countries is poor. This is not only the case in eastern and southern European countries. Also in northern countries like Germany, Denmark, Sweden and so on the medical care for these people is inadequate.

Some countries have specialist support for mainstream health services: in Finland and in The Netherlands there is a specialty -physician for people with intellectual disabilities. But in these countries these specialists are not easily available, and general practitioners are not always willing or able to provide adequate care. In the UK the Learning Disability Teams form a good design for good health care. However, we heard that in the beginning, financial and personal means were almost endless, but in recent years there has been a reduction of means. Another question in this country is whether the attention for the specific somatic aspects of care is sufficient.
This raised in 2002 the question within MAMH whether we could formulate standards of health care for people with intellectual disabilities that are valuable for every country, independently of their health care system. We decided to start a process which led to the finalising of these standards in November 2003. At that moment, in The Netherlands the first doctors finished their specialization to physician for people with intellectual disabilities, three years after the start of the training course for this new specialty.

A consortium of several Dutch organizations, with the Society of Physicians for People with Intellectual Disabilities (NVAVG) in the Netherlands playing a central role, initiated the process of preparing the manifesto.

The organizing committee formulated five basic criteria for adequate health care of people with intellectual disabilities. A wide coordinated process took place to explore and investigate upon these basic criteria.

Governmental reports, literature, and views gathered by this committee and by means of a thoroughly prepared questionnaire distributed to experts in different countries, were collected and studied. This phase lasted about one year.

In November 2003 an invitational conference organized by the Dutch Society of Physicians for Persons with Intellectual Disabilities (NVAVG), the Erasmus Medical Centre (Rotterdam) and MAMH took place in Rotterdam. The conference represented the final and consensus making stage in formulating and accepting the manifesto.

Five basic standards were formulated:

1. Optimal availability and accessibility to mainstream health services with primary care physicians playing a central role. This means that people with intellectual disabilities will:

a. Use mainstream health services.

b. Receive more time for consultations in the clinic or in home visits, when needed.

c. Receive adequate support in communication, when needed.

d. Receive a proactive approach to their health needs.

e. Have no extra financial, physical or legislative barriers to use mainstream services.

f. Be able to participate in screening programmes, in the same way as anybody else.

g. Be supported in achieving and maintaining a healthy lifestyle that will prevent illness and encourage positive health outcomes.

h. Receive understandable information about health and health promotion (also available to family and carers). 
i. Receive health care with good co-operation and co-ordination between different professionals.

2. Health professionals (especially physicians, psychiatrists, dentists, nurses and allied professionals) in mainstream health services will have competencies in intellectual disabilities and therefore in some of the more specific health problems in people with intellectual disabilities. This will require that:

a. Health professionals have a responsibility to achieve competencies in the basic standards of health care for people with intellectual disabilities.

b. These competencies include the awareness that not all the health problems of people with intellectual disability are caused by their disability.

c. All training programs for health professionals pay attention to intellectual disabilities, including the most common aetiology, some frequent syndromes, aetiology-related health problems, communication, legal and ethical aspects.

d. Training in attitude and communicational skills is as important as clinical skills and therefore is part of the training programs.

e. Guidelines on specific health issues are available through Internet, CD-ROM or otherwise.

f. Health care professionals in mainstream services have easy access to and are able to get advice from specialist colleagues without extra financial, practical or legislative barriers.

3. Health professionals (physicians, psychiatrists, dentists, nurses and allied professionals) who are specialised in the specific health needs of individuals with intellectual disabilities are available as a back-up to mainstream health services. These professionals can advise, treat specific medical problems or take over (a part of) the medical care for people with intellectual disabilities. This will require that:

a. Training Programmes are available for health professionals who want to gain competencies in health issues of people with intellectual disabilities.

b. These specialists create and maintain networks with specialised colleagues in and outside of their own profession, in order to improve their knowledge and skills. This can be achieved by personal contacts or by creating (virtual) centres of expertise. c. Research on health issues of people with intellectual disabilities is stimulated in co-operation with academic centres. Academic Chairs in Intellectual Disability Medicine should be created to initiate, stimulate and co-ordinate research projects.

4. Health care for individuals with intellectual disabilities often needs a multidisciplinary approach.

a. Specific health assessments and / or treatments need co-ordination between different health professionals (for example visual and hearing impairment, mental health care, care for people with multiple and complex disabilities, care for the elderly, rehabilitation care).

b. Specialist training for nurses and other carers is stimulated. This includes learning how to support and care for people with intellectual disabilities who have for instance sensory impairments, autistic spectrum disorders, epilepsy, mental health problems, behavioural/forensic problems, physical and complex disabilities, swallowing and feeding problems and age related problems.

5. Health care for people with intellectual disabilities needs a pro-active approach.

a. Participation in national screening programmes should be encouraged.

b. Anticipating health investigations on visual and hearing impairments and other frequent health problems should be evidence based and routinely available.

c. General and specific health monitoring programmes are developed and implemented. In the development of Health Indicator Systems special attention is paid to people with intellectual disabilities.

d. Responsibility for the development of anticipating investigation programmes and for their implementation must be clarified (primary care physicians, Public Health Doctors or specialised physicians).

e. People with intellectual disabilities and their families have a right to aetiological investigations.

This manifesto was translated in several languages and published in several international and national journals. It is a guiding document for developments in The Netherlands. In Scandinavian countries special care for people with intellectual disabilities seems to be a malediction. Normalisation and inclusion are in 
official politics translated there as "the same care as for everybody else". In those countries together with the de-concentration or de-institutionalisation the specific knowledge is evaporated. This is especially the case in Norway, Sweden and Denmark. Physicians there use the Manifesto as an internationally accepted document to put pressure on their governments.

The Manifesto is now, five years later, still a living and fertile document.

The Manifesto points out that there should be specialized health professionals, but also that general practitioners and medical specialists should have more knowledge of intellectual disability and the medical and social aspects of it.

We want to work this point out.

\section{Round table in Maastricht about training of doctors in intellectual disability medicine}

Therefore, in August 2006, MAMH organised together with IASSID-Europe, a Round Table conference about education of doctors in intellectual disability medicine. The central question was: what should medical students learn about intellectual disability and intellectually disabled people.

We have sent a questionnaire to doctors in several countries. Responses came from six European countries (Norway, Sweden, Finland, The Netherlands, Germany and the UK) and from the USA, Japan, Canada and Australia.

Attention for the specific aspects of intellectual disability medicine in the total educational programme for medical students varies from zero to 36 hours. In most countries there is no specific attention, just some general information spread over psychiatry, paediatrics and genetics.
In some countries there is a block -intellectual disability medicine- as an optional course.

All responses on what every doctor should know were similar on the main topics: doctors have to know people with intellectual disabilities, therefore you need to meet them, the importance of communication, different co-morbidity, aetiology, psychological aspects, ethical and legislative issues.

Also the support of parents of people with intellectual disabilities, preventive health care and population screening were frequently mentioned.

The round table decided to form a task force. This group has to establish a consensus document which can be used to put pressure on universities to implement more modules of intellectual disability medicine in their training courses for doctors.

For this document the following points are relevant:

1. Why is training in ID-medicine necessary? (health inequities and disparities)

2. A set up for the educational objectives to cover this need.

3. A plan for implementation in universities, medical colleges and society, including presentation to governments and parents organizations.

This activity seems to be, at least is meant to be, a logic next step after the European Manifesto.

We think that the results of these activities are indeed useful in all countries. However, we have to accept that developments in every single country have their own tempo, dependent of socio-cultural and financial circumstances. 